\title{
Mistakes that affect others: An fMRI study on processing of own errors in a social context
}

\author{
Sina Radke $\cdot$ F. P. de Lange $\cdot$ M. Ullsperger $\cdot$ \\ E. R. A. de Bruijn
}

Received: 13 December 2010 / Accepted: 1 April 2011 / Published online: 17 April 2011

(C) The Author(s) 2011. This article is published with open access at Springerlink.com

\begin{abstract}
In social contexts, errors have a special significance and often bear consequences for others. Thinking about others and drawing social inferences in interpersonal games engages the mentalizing system. We used neuroimaging to investigate the differences in brain activations between errors that affect only agents themselves and errors that additionally influence the payoffs of interaction partners. Activation in posterior medial frontal cortex (pMFC) and bilateral insula was increased for all errors, whereas errors that implied consequences for others specifically activated medial prefrontal cortex (mPFC), an important part of the mentalizing system. The results demonstrate that performance monitoring in social contexts involves additional processes and brain structures compared with individual performance monitoring where errors only have consequences for the person committing them. Taking into account how one's behavior may affect others is particularly crucial for adapting behavior in interpersonal interactions and joint action.
\end{abstract}

Keywords Error-monitoring $\cdot$ Mentalizing $\cdot$ Medial frontal cortex $\cdot$ Cingulate cortex $\cdot$ Social neuroscience . Perspective-taking

\section{Introduction}

Performance monitoring is crucial for successful goaldirected behavior. After commission of an error, preventing

S. Radke $(\bowtie) \cdot$ F. P. de Lange $\cdot$ M. Ullsperger $\cdot$ E. R. A. de Bruijn Donders Institute for Brain, Cognition and Behaviour, Radboud University Nijmegen, P.O. Box 9104, 6500 HE Nijmegen, The Netherlands

e-mail: s.radke@donders.ru.nl future errors and their negative consequences can be achieved by flexibly adapting one's behavior. Functional magnetic resonance imaging (fMRI) studies have demonstrated that posterior medial frontal cortex (pMFC) plays a crucial role in error-monitoring and subsequent behavioral adaptation (Ridderinkhof et al. 2004). PMFC involves midcingulate cortex (MCC) and pre-supplementary motor area (pre-SMA) and shows increased activation during error detection (De Bruijn et al. 2009). An electrophysiological correlate of this error-related brain activity is the errorrelated negativity (ERN), an event-related brain potential (ERP) that is generated after an erroneous response (Falkenstein et al. 1990; Gehring and Willoughby 2002) and negative feedback (Holroyd and Coles 2002). Coupling of fMRI and electroencephalography (EEG) has specified its origin being localized in the rostral cingulate zone (RCZ) of pMFC (Debener et al. 2005). The anterior insula is modulated by error awareness and has shown to be more active during errors of which one was aware compared with unaware errors (Ullsperger et al. 2010; Klein et al. 2007; Hester et al. 2005). Outcomes that signal a failure to attain the intended goal may give rise to affective and motivational responses to errors (Gehring and Willoughby 2002; Luu et al. 2000).

Both ERP and fMRI studies show that the detection of others' errors relies on similar neural mechanisms as own error-processing (van Schie et al. 2004; De Bruijn et al. 2009; Shane et al. 2009). Monitoring the actions of others is relevant in a variety of social interactions, especially in joint action, thus when coordinating actions in order to achieve a common goal. When acting together, a failure of one person may affect the interaction partner as well, e.g., most drastically by precluding the common goal to be reached, but also simply by altering the motivation of the other to continue working on the task. In real life, errors are 
often made within a social context and therefore have a special significance. An incorrect performance may yield negative consequences not only for agents themselves but also for other persons involved in the situation. These consequences may be evident, e.g., in material outcomes and reputation. A very explicit situation is a team sport like soccer when, for instance, missing a penalty kick affects not only the particular player who has missed it but also the team as a whole with regard to the final score. This may even extend to their reputation and the perceived competence of their coach, who, in turn, may face another cascade of negative consequences that starts with being fired, might go on with family members not meeting the expenses for certain costs of living anymore, etc.

However, taking into account how one's actions might affect others occurs almost automatically in daily life and often serves as an important guideline for decisionmaking. Although there are various everyday life situations where errors imply consequences for others, up to now, error-monitoring research has focused on errors that affect only the individual who has committed them. Although some studies investigating neural correlates of action observation include manipulations in such a way that actions of an agent have consequences for the observer, the focus usually lies on the observer and not the individual who is performing and therefore responsible for the consequences (De Bruijn et al. 2009; Marco-Pallares et al. 2010).

On the contrary, social inferences, i.e., thinking about others (first level) and reflecting about what others think about oneself (second level), have been extensively investigated within the last decade, especially from a social neuroscience perspective, and pertain to higher-level cognitive processes (Ochsner et al. 2005). Anticipating others' actions or reasoning about triadic relations (relations between two minds and an object; Saxe 2006) entails activation of the mentalizing system (Van Overwalle 2010). Neuroimaging studies have shown that the mentalizing system involves parietal cortex, temporoparietal junction (TPJ), temporal pole, and medial prefrontal cortex (mPFC) (Van Overwalle and Baetens 2009; Frith and Frith 2003). Of these, mPFC is the area of greatest relevance to "online" mentalizing during interactive paradigms (Assaf et al. 2009). According to the distinction of Van Overwalle (2010), mPFC is located between 30 and $60 \mathrm{~mm}$ on the $\mathrm{y}$-coordinate (posterior-anterior axis), whereas pMFC comprises the posterior part of the medial frontal cortex and is located between the 0 and $30 \mathrm{~mm}$ y-coordinate. This is in line with the theoretical model proposed by Amodio and Frith (2006), which subdivides medial frontal cortex (MFC) into anterior rostral MFC (arMFC; activated by social cognition tasks) and posterior rostral MFC (prMFC; activated by cognitive tasks) (Amodio and Frith 2006). Inferring traits and attributes about the self and others has been shown to selectively engage mPFC (Van Overwalle and Baetens 2009) of which especially the dorsal part is involved in spontaneous social-cognitive processing (Ma et al. 2010; Mitchell et al. 2006a). A further functional division of mPFC into a dorsal and a ventral subregion is proposed by Mitchell et al. (2006b) based on the perceived similarity of the other person: Whereas self-referencing tasks and mentalizing about similar others engage the ventral mPFC, dorsal mPFC activation is evident during mentalizing about dissimilar others. However, this differentiation does not reflect differences in valence (Mitchell et al. 2006b).

When committing an error also bears consequences for others, reflecting about their thoughts and other second level mentalizing processes are likely to be initiated. Therefore, social inferences about the scope and impact of one's errors on others may not be limited to activation of the error-monitoring network, but also engage areas related to mentalizing. Furthermore, the specific context might play a role as a competitive setting poses different mentalizing demands than a cooperative one and goes in hand with enhanced mPFC activation (Decety et al. 2004).

The aim of the current study was to investigate the differences in brain activity between errors that affect only the actors themselves and errors that also have consequences for others. The differential impact of errors was manipulated by using a cooperative versus a competitive setting that implemented distinctive payoffs for others. For erroneous compared with correct trials, activation in pMFC and insula should be evident irrespective of the particular setting and consequence, underlining their crucial role in error-monitoring. When comparing the context that implies consequences for others to the context that affects only the agents themselves, we expect engagement of areas related to mentalizing, such as parietal cortices and mPFC. Our main focus lies on the differences in brain activation between errors in the two contexts. We expect to find increased activation in mPFC for errors that additionally have an impact on others compared with errors that affect only the individual who has committed them. Although our manipulation of competition and cooperation differed from the one used by Decety et al. (2004), mPFC activation might further by modulated by the setting in such that a competitive, compared with a cooperative, setting entails higher activation of especially dorsal mPFC for errors that also affect an opponent than errors that only bear consequences for oneself. However, the findings by Mitchell et al. (2006a) do not suggest a differentiation in mPFC activity based on valence. 


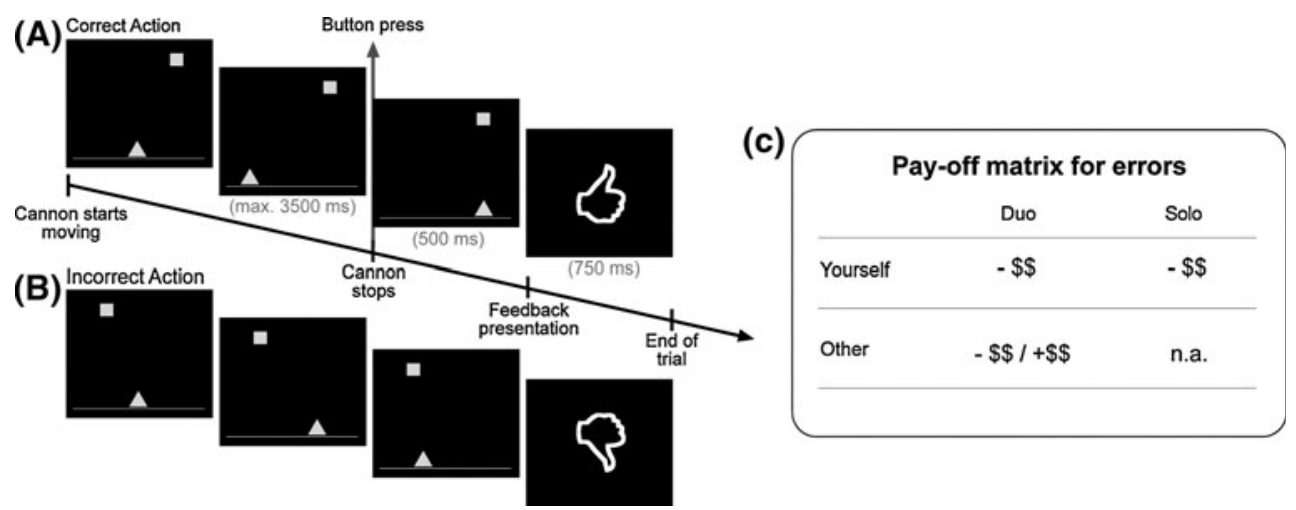

Fig. 1 Cannon shooting task and payoff matrix. Examples of a correct (a) and an incorrect action (b) are shown. fMRI was time-locked to the presentation of the feedback, always containing the crucial information about the correctness of the performed action. (c) Payoff matrix for the

\section{Methods}

Participants

The same subjects as in De Bruijn et al. (2009) were recruited. We measured brain activity in 34 healthy participants (16 female, mean age $=25.6$ years, $\mathrm{SD}=3$ years) who played a challenging computer game independently (solo) and in cooperation with or in competition against another person (duo). Of these, 20 participants ( 9 women, mean age $=26.2$ years, $\mathrm{SD}=3.2$ years) were assigned to a cooperative setting for the duo condition and 14 participants ( 7 women, mean age $=24.7$ years, $\mathrm{SD}=2.4$ years) were assigned to a competitive one. Note that a different analysis of the data from the duo session addressing a different functional question was already published in De Bruijn et al. (2009). Participants provided written informed consent prior to participation and the study was approved by the local research ethics committee.

Task and design

Participants performed a computerized task, the cannon shooting game (see Fig. 1). The aim of the task was to stop a horizontally moving cannon (triangle) by a button press, precisely lining it up with a stationary target in order to shoot the target (square). The size of the target was dynamically adapted based on participants' performance such that a mean hit rate of around $60 \%$ was achieved.

Participants started with a financial bonus of 5 Euro in both the solo condition and the competitive setting, while the cooperative setting was enforced by a bonus of 10 Euro. Committing an error was always associated with a monetary loss (10 cents) for the participant. In the duo condition, errors additionally bore consequences for the other player, either monetary loss (cooperative setting) or gain (competitive two conditions. Committing an error was always associated with a monetary loss (10 cents) for the participant. In the duo condition, errors additionally bore consequences for the other player, either monetary loss (cooperative setting) or gain (competitive setting; each 10 cents)

setting; each 10 cents). Correct responses had no effect on any player's bonus.

For each experimental session, two participants came into the lab and were introduced to each other. They received instructions together, after which one participant went to one scanner and the other participant to the other. They were told that during the duo condition the other player would see the same computer screen they saw while performing the task. Unknown to the real participant, the other participant was a confederate. The confederate was always the same person (a woman) throughout the entire experiment. Participants performed two blocks of the task, equivalent to the solo and the duo condition, with 100 trials each. The order of blocks was counterbalanced between subjects. Afterward, a questionnaire was administered outside the scanner to assess subjective ratings about, e.g., difficulty of the task, bonus motivation, or feelings, via a visual analog scale with a length of $100 \mathrm{~mm}$.

The original setup included additional conditions (i.e., observing others' performance) that allowed to disentangle brain activity of error and reward processing, which have been previously reported elsewhere in the context of observed error-processing in different settings (De Bruijn et al. 2009).

Stimulus presentation was controlled with Presentation software (Neurobehavioral Systems, Albany, NY, USA). The onset of the trial was jittered with a blank screen for $0,250,500,750$, or $1,000 \mathrm{~ms}$. Null events were added to allow the blood-oxygenation level-dependent (BOLD) response to go back to baseline. A trial started with the presentation of the target and the cannon. Target location was randomly determined on each trial, while the cannon was always horizontally centered. Immediately following presentation, the cannon started moving either to the right or to the left for a maximum of 2.5 lengths 
$(3,500 \mathrm{~ms})$ in total. An unambiguous feedback signal (thumb up/thumb down) was presented $500 \mathrm{~ms}$ following the button press, indicating whether the response resulted in a hit or a miss.

\section{Data acquisition}

Imaging was performed at 3T on a Siemens Trio scanner (Siemens, Erlangen, Germany) at the Max Planck Institute for Human Cognitive and Brain Sciences, Leipzig, Germany. Twenty-eight functional slices were obtained parallel to the anterior commissure-posterior commissure (ACPC) line using a single-shot gradient echoplanar imaging (EPI) sequence (repetition time, $2 \mathrm{~s}$; echo time, $30 \mathrm{~ms}$; $64 \times 64$ pixel matrix; flip angle, $90^{\circ}$; field of view, $192 \mathrm{~mm}$ ) sensitive to blood-oxygen level-dependent contrast. Trials occurred at multiple, systematically offset time points (range 0-.5 s) in relation to the image acquisition in order to jitter the onset of the trials with respect to slice acquisition.

\section{MRI data analysis}

The fMRI data were preprocessed and analyzed in an event-related manner, within the general linear model, using SPM5 software (Wellcome Department of Imaging Neuroscience, London, UK). Preprocessing consisted of spatial realignment, slice-timing correction, normalization, and spatial smoothing with a Gaussian kernel with a full width at half-maximum of $5 \mathrm{~mm}$. At the first (subject) level, we used a single statistical linear regression model for all our analyses, as follows. Each trial was modeled as a boxcar, of which the onset corresponded to feedback onset and convolved with a standard hemodynamic response function (HRF). To control for effects of task difficulty, an additional regressor that modeled target size was included in the model. To correct for motion-related artifacts, subject-specific realignment parameters were modeled as covariates of no interest. Linear contrasts of regression coefficients (contrast images) were computed at the individual subject level and then subjected to a second-level random-effect analysis. Three paired $t$ Tests with an uncorrected voxel threshold of $P<.001 \quad(T=3.21)$, corrected for multiple comparisons at the cluster level $(P<.005)$, were computed to reveal the differences between erroneous and correct responses, between taskrelated activity in the solo and the duo context, and between error-related activity in the duo context and error-related activity in the solo context. In order to investigate the between-subject effect of setting (cooperative, competitive), contrast images of the comparison error(duo) $>$ correct(duo) were computed on the first level for each setting. These contrast images were then subjected to the second level where a 2-sample $t$ Test was performed with an uncorrected voxel threshold of $P<.001(T=3.36)$, corrected for multiple comparisons at the cluster level $(P<.005)$ (Friston et al. 1996).

\section{Results}

Behavioral results and manipulation check

In the solo condition, the average hit rate was $63.2 \%$ $(\mathrm{SD}=5.1 \%)$. In the duo condition, the average hit rate was $63.0 \%(\mathrm{SD}=4.8 \%)$ in the cooperative and $63.7 \%$ $(\mathrm{SD}=2.7 \%)$ in the competitive setting, respectively. A repeated-measures ANOVA demonstrated that performance did neither differ between solo and duo nor between cooperation and competition (all $p \mathrm{~s}>.60$ ), which verifies that adapting the size of the target based on participants' performance was successful. Similarly, mean ratings on task difficulty did not differ between the duo and the solo condition $(M=46.3 \mathrm{~mm}$ and $M=46.1 \mathrm{~mm}$, respectively; $P=.95)$ nor between the cooperative and the competitive setting $\left(M_{\text {coop-duo }}=44.9 \mathrm{~mm}, M_{\text {comp-duo }}=47.93 \mathrm{~mm}, P=.55\right.$; $\left.M_{\text {coop-solo }}=43.8 \mathrm{~mm}, M_{\text {comp-solo }}=50.0 \mathrm{~mm}, P=.34\right)$. Moreover, there were no differences in reaction times between correct and erroneous responses, between solo and duo consequences, and between the cooperative and the competitive setting (all $p \mathrm{~s}>.32$ ).

The exit questionnaire revealed that participants were aware that their errors affected the other player in the duo condition as ratings significantly differed from 0 $(M=40.7 \mathrm{~mm}, t=8.09, d f=33, P<.0001$; no difference between the cooperative and competitive setting: $M_{\text {coop }}=$ $38.0 \mathrm{~mm}, M_{\text {comp }}=44.5 \mathrm{~mm}, P=.53$ ). However, participants were more annoyed by their own errors in the duo condition than in the solo condition $(M=63.7 \mathrm{~mm}$ and $M=58.6 \mathrm{~mm}$, respectively; $t=1.75, d f=33, P=.045$, one-tailed), which also did not differ between settings $\left(M_{\text {coop-duo }}=61.3 \mathrm{~mm}, \quad M_{\text {comp-duo }}=67.1 \mathrm{~mm}, \quad P=.46\right.$; $\left.M_{\text {coop-solo }}=57.2 \mathrm{~mm}, M_{\text {comp-solo }}=60.6 \mathrm{~mm}, P=.66\right)$.

\section{FMRI results}

As expected, the main effect of response type (erroneous compared with correct responses) showed activation in the error-monitoring network, including $\operatorname{pMFC}(4,22,36)$ and bilateral insula $(36,20,2 ;-42,18,-10)$, and was independent of setting and consequence (see Table 1 and Fig. 2).

When comparing overall task-related activity in the context that implied consequences for others to overall taskrelated activity in the condition that concerned only the 
Table 1 Spatial coordinates of the local maxima of brain regions showing significant effects in the different contrasts

\begin{tabular}{|c|c|c|c|c|c|}
\hline Contrast & Anatomical region & $t$ value & Cluster size & $P$ value & $\begin{array}{l}\text { Stereotactic } \\
\text { coordinates }(\mathrm{x}, \mathrm{y}, \mathrm{z})\end{array}$ \\
\hline \multirow[t]{3}{*}{ Error $>$ correct } & $\begin{array}{l}\text { (R) posterior medial } \\
\text { frontal cortex }\end{array}$ & 9.13 & 1,025 & $<.001$ & $4,22,36$ \\
\hline & $\mathrm{R}$ insula & 8.85 & 902 & $<.001$ & $36,20,2$ \\
\hline & $\mathrm{L}$ insula & 6.48 & 632 & $<.001$ & $-42,18,-10$ \\
\hline \multirow[t]{4}{*}{ Duo $>$ solo } & L posterior parietal cortex & 7.41 & 249 & $<.001$ & $-34,-68,52$ \\
\hline & $\mathrm{R}$ posterior parietal cortex & 7.41 & 144 & $<.001$ & $42,-66,48$ \\
\hline & (R) medial prefrontal cortex & 6.26 & 373 & $<.001$ & $10,38,24$ \\
\hline & L temporal pole & 5.89 & 167 & $<.001$ & $-42,14,-16$ \\
\hline $\begin{array}{c}\text { Correctness } \times \text { consequence } \\
(\text { Error }>\text { correct }(\text { duo })> \\
\text { Error }>\text { correct }(\text { solo }))\end{array}$ & L medial prefrontal cortex & 6.98 & 439 & $<.001$ & $-10,46,42$ \\
\hline
\end{tabular}

Stereotactic coordinates correspond to the standard Montreal Neurological institute (MNI) brain

Fig. 2 Sagittal and coronal view of error-specific brain activations in both contexts (duo and solo). Increased activation in $\operatorname{pMFC}(4,22,36)$ and bilateral insula $(36,20,2 ;-42,18,-10)$ was evident for erroneous compared with correct responses. Thresholded at $T=3.36$, corrected for multiple comparisons at the cluster level

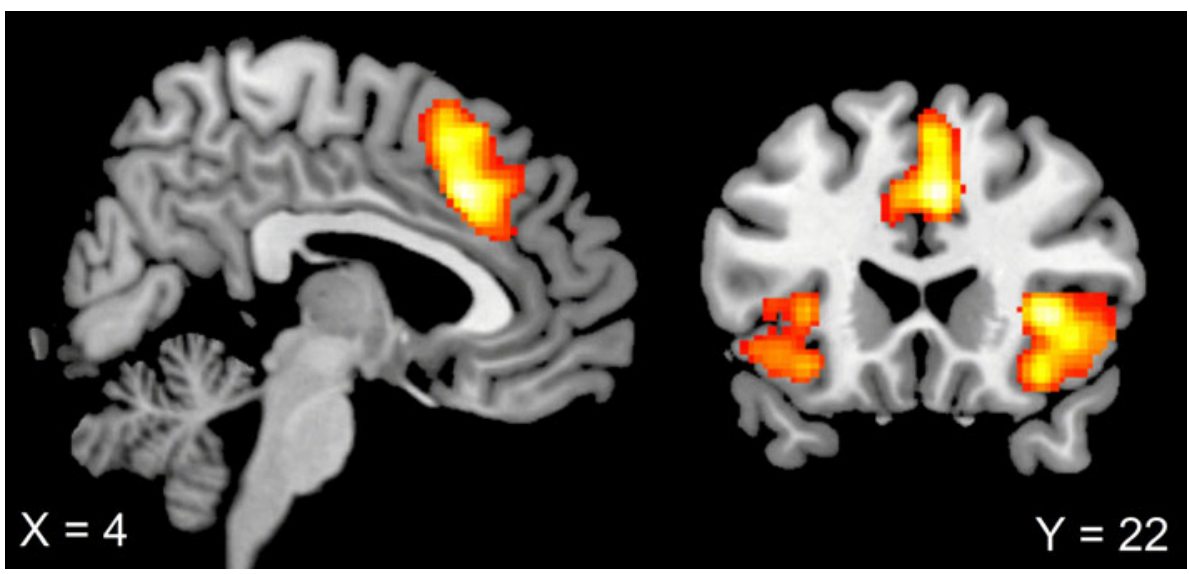

Fig. 3 Coronal and sagittal view of context-related brain activations. Increased activation in posterior parietal cortices $(-34,-68,52 ; 42,-66,48)$, $\operatorname{mPFC}(10,38,24)$, and the left temporal pole $(-42,14,-16)$ was evident for the context which implied consequences for others (duo) compared with the condition that affected only the agents themselves. Thresholded at $T=3.36$, corrected for multiple comparisons at the cluster level

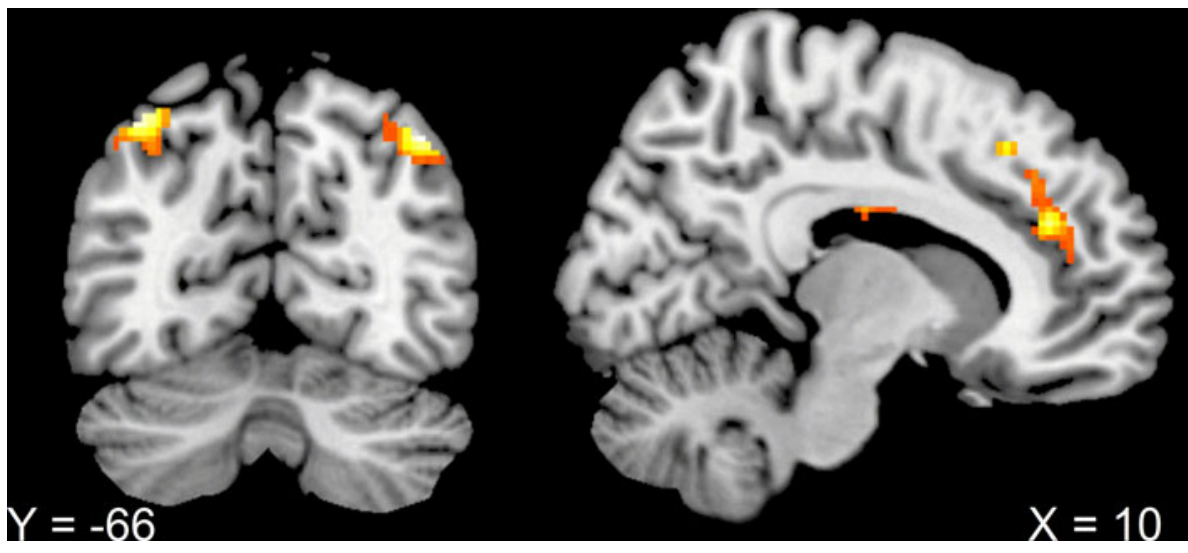

agents themselves (duo vs. solo), there was larger activation for the duo context in the posterior parietal cortices $(-34,-68,52 ; 42,-66,48), \operatorname{mPFC}(10,38,24)$ and the left temporal pole $(-42,14,-16)$ (see Fig. 3).

When comparing error-related activity in the duo context to error-related activity in the solo context, enhanced activity in $\mathrm{mPFC}(-10,46,42)$, extending to the left superior frontal gyrus, was evident (see Fig. 4).
The comparison of the two settings in the duo condition (cooperation vs. competition and the reverse comparison) did not reveal any clusters above threshold. ${ }^{1}$

\footnotetext{
${ }^{1}$ In order to test whether the current fMRI results are affected by unequal sample sizes $(N=20$ vs. $N=14)$ we re-ran the analyzes with equal sample sizes of $N=14$ and did not find any differences in brain activation for any of the contrasts reported.
} 
Fig. 4 Sagittal and axial view of differential error- and contextrelated brain activations. Increased activation in $\mathrm{mPFC}$ $(-10,46,42)$ was evident for errors made in the context which implied consequences for others (duo) compared with errors committed in the condition that affected only the agents themselves (solo). Thresholded at $T=3.36$, corrected for multiple comparisons at the cluster level

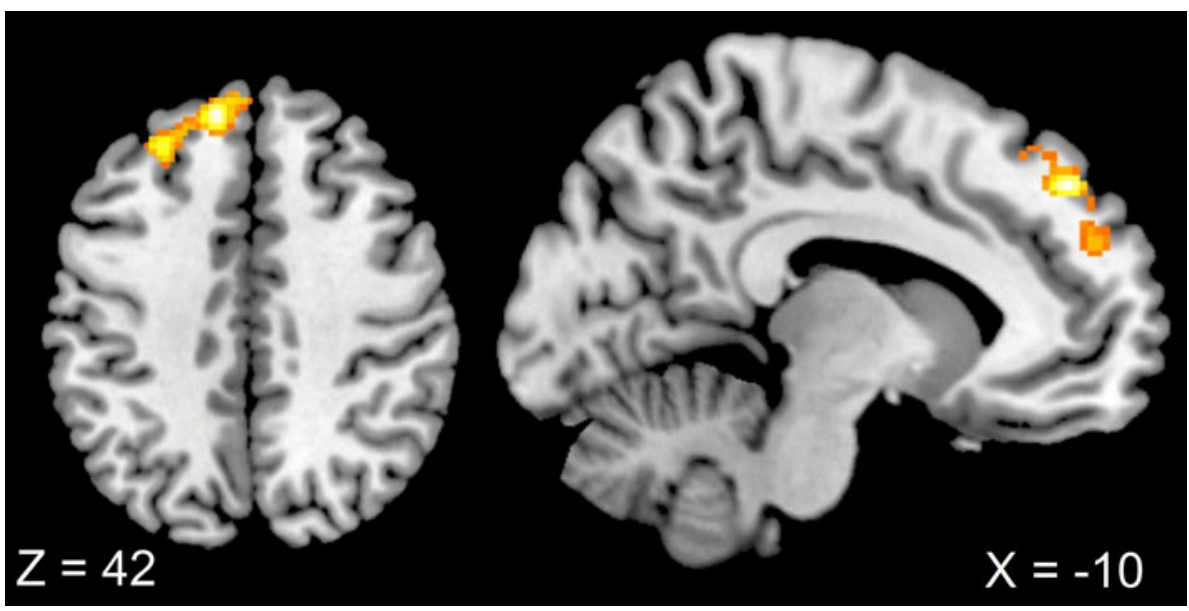

\section{Discussion}

The current study focused on performance monitoring in a social context by investigating the differences in brain activations between errors that affect only agents themselves and errors that also have consequences for others. The error-related activations in pMFC and the insula are in line with previous findings (e.g., De Bruijn et al. 2009; Ridderinkhof et al. 2004) and occurred independently of the consequence of one's errors, i.e., whether the monetary consequences were restricted to the agents themselves or whether errors also affected the other players' bonus, and also independent of the setting, i.e., whether one's errors resulted in monetary gain or loss with regard to the partner/ competitor. Similarly, activation of parietal cortices and temporal poles has been previously reported with regard to interactive games and Theory of Mind paradigms (Decety et al. 2004; Olson et al. 2007). However, errors that implied consequences for others specifically activated mPFC, an important part of the mentalizing system (Frith and Frith 2003). Although neither thinking about other players nor actually interacting with them was explicitly required for performing the task, participants most likely engaged in social reasoning as reflected by $\mathrm{mPFC}$ activity.

\section{Engagement of the mentalizing system}

When participants were aware of the fact that their actions also had consequences for others, activation in temporal pole, parietal cortex, and mPFC was evident. These areas constitute the mentalizing system (Frith and Frith 2003) and are likely to be responsible for integrating social information in a particular context. Especially the temporal poles are regarded as convergence zones of all sensory modalities (Damasio et al. 2004) that enable us to develop both general knowledge and momentarily conclusions about a particular person in a particular situation. In the context of mentaliz- ing, Frith and Frith (2003) argue that the temporal poles generate a broad semantic and emotional framework which facilitates processing of the current input. They might also play a role in retrieving information about social schemas and scripts, which helps "simulating" the experiences of others and anticipating their goals (Gallagher and Frith 2003). A comparable role can be attributed to the posterior parietal cortices which are involved in the sense of agency, i.e., referring to the agent of an action and monitoring whether self-produced actions are in accordance with their consequences (David et al. 2008). Likewise, Decety et al. (2004) report engagement of the posterior parietal cortices during cooperation as well as during competition. The independence of posterior parietal involvement from the specific mental set is supported by the overall task-related activity that was evident in the current study.

Moreover, activation in mPFC was enhanced when actions affected not only the agents themselves, but also other players involved in the game situation. This was observable both in the rather "tonic" mentalizing effect which was independent of the actual correctness of an action, reflecting larger activation when another person is potentially affected than when playing alone, and particularly when contrasting error trials that do and do not have consequences for others. Whereas the former might indicate mentalizing processes that operate relatively automatically in the background during the whole course of the duo condition, the strong $\mathrm{mPFC}$ activation in response to errors can be explained by our design: Importantly, correct responses did not have an effect on any player's score; only errors altered their bonus. Crucially, this was implemented through the payoff structure that linked errors as specific actions to monetary consequences for participants. Although committing an error always entailed a monetary loss for oneself, whether playing alone or in a cooperative or competitive setting, participants were more annoyed by errors made in the duo condition than in the solo condition. 
This suggests that these errors indeed had a special significance and stimulated mentalizing processes.

Implict and explicit mentalizing

Both settings, cooperation and competition, entailed a common activation pattern in $\mathrm{mPFC}$, which reflects rather general aspects of mentalizing and contradicts with the context-specific demands suggested by Decety et al. (2004). However, in our task, mentalizing takes place in both settings and independently of the actual mental/affective state of the other associated with the committed error. Note that contrary to Decety et al.'s (2004), but similar to Mitchell et al.'s (2006a) study, anticipating possible actions of a partner or opponent was not part of the task. In previous experiments, mentalizing has often been implemented rather explicitly by instructing participants to infer or judge the mental states of others, including fictional characters and animated shapes (Van Overwalle and Baetens 2009). In contrast, we used a design that involves implicit mentalizing as participants were not required to think about the other participants and their intentions or actions. It has been shown that the core mentalizing areas, especially mPFC, are recruited during impression formation and spontaneous processing of social information, thus without the deliberate endeavor to make social judgments (Ma et al. 2010; Mitchell et al. 2006a).

\section{Performance-monitoring perspective}

A study by Hajcak et al. (2005) manipulated the motivational significance of errors. When participants thought that their performance was being evaluated by a person sitting next to them, the ERN in response to committing errors was larger. The authors argue that an error is more salient and significant when subjects are confronted with the prospect of receiving evaluative feedback about their performance (Hajcak et al. 2005). Along with the evaluation, participants were also observed while performing the task, which is similar to our current paradigm and may lead to concerns and worries about one's reputation-a process that fundamentally depends on mentalizing. However, in Hajcak et al.'s design, errors only affected the actors' own reputation; thus there were only negative consequences for participants themselves without any implications for the evaluators, whereas our central manipulation was that errors also bore consequences for others. Koban et al. (2010) investigated the impact of the social context (cooperation vs. competition) on brain potentials and found no differences in the ERN between contexts when participants were observed by their competitor or partner. However, their design also lacked the direct consequences for both oneself and the other player since only overall performance (in two blocks, not trial-wise) determined the outcomes (Koban et al. 2010). Previously published data (De Bruijn et al. 2009) indicated that activity in pMFC was increased for all types of errors, regardless of setting and agency. Yet, the activation we found is located more anterior than the usually reported generator of the ERN (Debener et al. 2005), which is more in line with its role in social inferences and mentalizing.

\section{Limitations}

The asymmetrical manipulation that only errors, but not correct responses, have consequences for oneself and others might not be ideal under methodological considerations. As errors were always associated with a negative outcome in the current design, no inferences about reward processing in terms of positive valences can be made. A previous study addressed this topic in detail (De Bruijn et al. 2009) and demonstrated an effect of setting for striatal, but not for medial frontal areas. As our focus was not on reward-related processes, but on contrasting brain activity between errors that affect only the actors themselves and errors that also have consequences for others, we chose not to reward correct actions either way (neither for oneself nor others). However, our results do indicate that mentalizing does not depend on the valence of an error for the other player (i.e., whether it entailed a monetary loss or gain).

Moreover, it would be helpful to include standard Theory of Mind paradigms as well as control tasks in future studies in order to assess the overlap between different paradigms, i.e., explicit and implicit mentalizing tasks, preferably in within-subject designs.

\section{Differentiation between pMFC and $\mathrm{mPFC}$}

The recent meta-analysis of Van Overwalle (2010) proposes a distinction between pMFC and mPFC based on both coordinates and function: Whereas the pMFC (between 0 and $30 \mathrm{~mm}$ on the $\mathrm{y}$-coordinate) is involved in conflict- and error-processing, the mPFC (between 30 and $60 \mathrm{~mm}$ ) is recruited during mentalizing and social reasoning (Van Overwalle 2010). Our results underline the differentiation between pMFC and mPFC nicely by disentangling brain activity that is related to error-processing from social inferences pertaining to the consequences that one's errors imply for others.

\section{Conclusion}

To conclude, the current study was the first to investigate the neural mechanisms involved in processing own errors 
that also entail consequences for an interaction partner. The results demonstrate that performance monitoring in social contexts engages additional processes and brain structures compared with individual performance monitoring where errors only have consequences for the person committing them. When bearing in mind how one's own actions affect others, mentalizing plays an important role. Performance monitoring in social contexts is likely to be a highly complex process that becomes increasingly difficult when outcomes are uncertain or ambiguous, as often in real life. This is particularly evident in symptoms of obsessive-compulsive disorder, i.e., intrusions that one's actions will harm others, especially loved ones, and the arising fear of taking the wrong action. Further research in both healthy and psychiatric populations might elucidate how the relationship between the agent and other individuals involved could modulate these processes and their potential disturbance. Psychiatric patients with clear social deficits may show impaired performance monitoring in social contexts, while this may not be apparent when looking at individual performance monitoring. For patients with depression, additional factors like negative internal attributions and self-blame or the experience of uncontrollability might lead to an alteration of behavior in different social contexts where errors have a special significance.

Acknowledgments EDB was supported by a VENI grant from the Netherlands Organization for Scientific Research (NWO) (451-07022). The authors report no financial interests or any conflicts of interest. The authors thank Mareike Menz and the technical staff at the MPI in Leipzig for their support in data collection.

Open Access This article is distributed under the terms of the Creative Commons Attribution Noncommercial License which permits any noncommercial use, distribution, and reproduction in any medium, provided the original author(s) and source are credited.

\section{References}

Amodio DM, Frith CD (2006) Meeting of minds: the medial frontal cortex and social cognition. Nat Rev Neurosci 7(4):268-277

Assaf M, Kahn I, Pearlson GD, Johnson MR, Yeshurun Y, Calhoun VD, Hendler T (2009) Brain activity dissociates mentalization from motivation during an interpersonal competitive game. Brain Imaging Behav 3(1):24-37

Damasio H, Tranel D, Grabowski T, Adolphs R, Damasio A (2004) Neural systems behind word and concept retrieval. Cognition 92(1-2):179-229

David N, Newen A, Vogeley K (2008) The "sense of agency" and its underlying cognitive and neural mechanisms. Conscious Cogn 17(2):523-534

De Bruijn ERA, de Lange FP, von Cramon DY, Ullsperger M (2009) When errors are rewarding. J Neurosci 29(39):12183-12186

Debener S, Ullsperger M, Siegel M, Fiehler K, von Cramon DY, Engel AK (2005) Trial-by-trial coupling of concurrent electroencephalogram and functional magnetic resonance imaging identifies the dynamics of performance monitoring. J Neurosci 25(50):11730-11737
Decety J, Jackson PL, Sommerville JA, Chaminade T, Meltzoff AN (2004) The neural bases of cooperation and competition: an fMRI investigation. Neuroimage 23(2):744-751

Falkenstein M, Hohnsbein J, Hoormann J, Blanke L (1990) Effects of errors in choice reaction tasks on the ERP under focused and divided attention. In: Brunia CHM, Gaillard AWK, Kok A (eds) Psychophysiological brain research. Tilburg University Press, Tilburg, pp 192-195

Friston KJ, Holmes A, Poline JB, Price CJ, Frith CD (1996) Detecting activations in PET and fMRI: Levels of inference and power. Neuroimage 4(3):223-235

Frith C, Frith U (2003) Development and neurophysiology of mentalizing. Philos T Roy Soc B 358:459-473

Gallagher HL, Frith CD (2003) Functional imaging of 'theory of mind'. Trends Cogn Sci 7(2):77-83

Gehring WJ, Willoughby AR (2002) The medial frontal cortex and the rapid processing of monetary gains and losses. Science 295(5563):2279-2282

Hajcak G, Moser JS, Yeung N, Simons RF (2005) On the ERN and the significance of errors. Psychophysiology 42(2):151-160

Hester R, Foxe JJ, Molholm S, Shpaner M, Garavan H (2005) Neural mechanisms involved in error processing: A comparison of errors made with and without awareness. Neuroimage 27(3): 602-608

Holroyd CB, Coles MGH (2002) The neural basis of human error processing: Reinforcement learning, dopamine, and the error-related negativity. Psychol Rev 109(4):679-709

Klein TA, Endrass T, Kathmann N, Neumann J, von Cramon DY, Ul1sperger M (2007) Neural correlates of error awareness. Neuroimage 34(4): 1774-1781

Koban L, Pourtois G, Vocat R, Vuilleumier P (2010) When your errors make me lose or win: Event-related potentials to observed errors of cooperators and competitors. Soc Neurosci 5(4):360-374

Luu P, Flaisch T, Tucker DM (2000) Medial frontal cortex in action monitoring. J Neurosci 20(1):464-469

Ma N, Vandekerckhove M, Van Overwalle F, Seurinck R, Fias W (2010) Spontaneous and intentional trait inferences recruit a common mentalizing network to a different degree: Spontaneous inferences activate only its core areas. Soc Neurosci 21:1-16

Marco-Pallares J, Kramer UM, Strehl S, Schroder A, Munte TF (2010) When decisions of others matter to me: an electrophysiological analysis. BMC Neurosci 11(86)

Mitchell JP, Cloutier J, Banaji MR, Macrae CN (2006a) Medial prefrontal dissociations during processing of trait diagnostic and nondiagnostic person information. Soc Cog Aff Neurosci 1(1):49-55

Mitchell JP, Macrae CN, Banaji MR (2006b) Dissociable medial prefrontal contributions to judgments of similar and dissimilar others. Neuron 50(4):655-663

Ochsner KN, Beer JS, Robertson ER, Cooper JC, Gabrieli JDE, Kihsltrom JF, D'Esposito M (2005) The neural correlates of direct and reflected self-knowledge. Neuroimage 28(4):797-814

Olson IR, Plotzker A, Ezzyat Y (2007) The Enigmatic temporal pole: a review of findings on social and emotional processing. Brain 130(7):1718-1731

Ridderinkhof KR, Ullsperger M, Crone EA, Nieuwenhuis S (2004) The role of the medial frontal cortex in cognitive control. Science 306:443-447

Saxe R (2006) Uniquely human social cognition. Curr Opin Neurobiol 16(2):235-239

Shane MS, Stevens MC, Harenski CL, Kiehl KA (2009) Double dissociation between perspective-taking and empathic-concern as predictors of hemodynamic response to another's mistakes. Soc Cogn Aff Neurosci 4(2):111-118

Ullsperger M, Harsay HA, Wessel JR, Ridderinkhof KR (2010) Conscious perception of errors and its relation to the anterior insula. Brain Struct Funct 214:629-643 
Van Overwalle F (2010) A dissociation between social mentalizing and general reasoning. NeuroImage. doi:10.1016/j.neuroimage. 2010.1009.1043

Van Overwalle F, Baetens K (2009) Understanding others' actions and goals by mirror and mentalizing systems: A meta-analysis. Neuroimage 48(3):564-584 van Schie HT, Mars RB, Coles MGH, Bekkering H (2004) Modulation of activity in medial frontal and motor cortices during error observation. Nat Neurosci 7(5):549-554 